\title{
Relación entre calidad de vida, tiempo de consumo y tiempo de permanencia de los niños, niñas y adolescentes adictos a sustancias psicoactivas en una comunidad terapéutica de Lima
}

\begin{abstract}
Relationship between health-related quality of life (HRQL), length of consumption of psychoactive substances, and length of stay of children and adolescents (males and females) in a therapeutic community in Lima (Peru).
\end{abstract}

\section{Elena Esther Yaya Castañeda ${ }^{1}$}

\section{RESUMEN}

El presente estudio tuvo como objetivo determinar la relación entre la calidad de vida relacionada con la salud (CVRS), el tiempo de consumo de sustancias psicoactivas y el tiempo de permanencia de niños, niñas y adolescentes en la una comunidad terapéutica de Lima (Perú). Se utilizó un cuestionario de datos del problema de consumo de sustancias psicoactivas de la comunidad terapéutica y un instrumento de evaluación de la calidad de vida en adictos a sustancias psicoactivas (TECVASP), que fue aplicado a 86 participantes, entre niños, niñas y adolescentes, de 7 a 18 años, de ambos sexos, de una comunidad terapéutica de Lima. La mayoría de los participantes (más del 70\%) reportaron un nivel bajo de CVRS. Asimismo, se hallaron correlaciones negativas entre la CVRS y tanto el tiempo de consumo como el tiempo de permanencia en la comunidad terapéutica. Se resalta la necesidad de realizar más estudios acerca de las repercusiones del consumo de SPA en la CVRS en poblaciones de este nivel de edad.

PALABRAS CLAVE: adolescentes, calidad de vida, niños, sustancias psicoactivas, tiempo de consumo, tiempo de permanencia

\section{SUMMARY}

This study aimed for determining the relationship between health-related quality of life (HRQL), length of consumption of psychoactive substances, and length of stay of children and adolescents (males and females) in a therapeutic community in Lima (Peru). A questionnaire of data on the problem of use of psychoactive substances of the therapeutic community and a questionnaire to evaluate the quality of life in addicts to psychoactive substances (TECVASP) were used, which were applied to 86 participants, among children and adolescents from 7 to 18 years old, of both sexes, from a therapeutic community in Lima. A most of participants (more than 70\%) reported a low level in quality of life. Furthermore, negative correlations were found between quality of life and length of consumption and length of stay in the therapeutic community. It is stressed the need of carrying out studies about impact of drug consumption in the quality of life in populations of this age.

KEY WORDS: Adolescents, quality of life, children, psychoactive substances, length of consumption, length of stay

\footnotetext{
Universidad Nacional Federico Villarreal. Lima, Perú.
} 


\section{INTRODUCCIÓN}

Los expertos del grupo World Health Organization Quality of Life (WHOQOL) partieron de la siguiente definición de CV (1995, p. 1405): la percepción de los individuos sobre su posición en la vida en el contexto de la cultura y el sistema de valores en el que viven, y con relación a sus metas, expectativas, normas y preocupaciones. Es un concepto amplio que oscila, incorporando de una manera compleja la salud física de las personas, el estado psicológico, el nivel de independencia, las relaciones sociales y su relación con los rasgos salientes de su ambiente.

Las revisiones teóricas y las investigaciones de calidad de vida relacionada a la salud (CVRS), en drogodependientes, refieren que la definición teórica está comprometida con el área física y psicosocial (Stuifbergen, Seraphine \& Roberts, 2000).

El área física, en la CVRS, incluye tres categorías presentes en la persona: el estado funcional, síntomas físicos y dependencia física. El estado funcional hace referencia a la capacidad de las personas de ejecutar una serie de actividades normales para la mayoría de las personas. Éste es reconocido como un indicador de relevancia social que puede propiciar un incremento en la imagen de deterioro de las personas en la CVRS. El dominio psicosocial está referido a la categoría de dependencia psicológica, la cual recoge información psicológica acerca de la necesidad de consumir drogas. En el dominio psicosocial se incluye una categoría sobre síntomas psicológicos, que difieren en su contenido según la alteración de salud de los pacientes. En el caso de la población consumidora de drogas, los síntomas que más frecuentemente son referidos son: depresión, ansiedad y agresividad. El deterioro del funcionamiento cognitivo es otra de las manifestaciones del consumo continuo de drogas. Las áreas cognitivas que se ven afectadas por el consumo de drogas son variadas y dependen, en buena parte, de la sustancia ingerida; estos problemas pueden ser de memoria, concentración, orientación y alucinaciones. Al hablar de percepción general de la salud se entiende la valoración que hace la persona respecto a en qué medida cree que el estado de su salud ha afectado a su vida cotidiana.

La categoría de aspectos sociales la definimos como la percepción de relaciones y contactos sociales que establece el individuo en su ambiente, estos pueden ser importantes en el entorno del individuo. Las expectativas de superar una enfermedad son experimentadas de manera muy positiva por los pacientes, las cuales pueden ser la base de la motivación, ya que se relacionan con la evaluación positiva de la CVRS. En el caso de la población consumidora de drogas, el fracaso en los intentos de abandonar las drogas influye de manera severa sobre las expectativas que éstos tienen, y a su vez sobre su CVRS. En este sentido, los drogodependientes experimentan sentimientos de culpa, frustración y desesperanza que se relacionan negativamente con la CVRS.

Haciendo una revisión de la literatura (e. g., Berzon, Hays \& Shumaker, 1993; Breslin, 1991; Corless, Nicholas \& Nokes, 2001; Wilson \& Cleary, 1995), podemos decir que en la actualidad se considera que calidad de vida, es un concepto multidimensional que engloba aspectos en las tres grandes esferas de la vida cotidiana: físicos, psicológicos y sociales.

Por otro lado, según la OMS (1969), la drogodependencia es el estado psíquico y físico causado por la acción recíproca entre un organismo vivo y un fármaco (droga), que se caracteriza por las modificaciones del comportamiento y por otras reacciones, que comprenden siempre un impulso irreprimible a tomar el fármaco (droga) en forma continua o periódica a fin de experimentar sus efectos psíquicos y, a veces, para evitar el malestar producido por la privación. Se trata de un síndrome manifestado por un patrón conductual donde el uso de una sustancia tiene más prioridad que otras conductas, lo cual lleva a establecer este trastorno como un impulso repetido a comprometerse en conductas poco productivas, una tensión creciente hasta que se realiza la conducta, y desaparición rápida de la tensión al realizarla.

Según la Comisión Nacional para el Desarrollo y Vida sin Drogas del Perú (Devida) (2013), el alcohol es la droga legal más consumida en el país por los escolares de secundaria de ambos sexos; la prevalencia de vida para esta sustancia fue de $37.2 \%$ y la edad promedio de inicio, 13.3 años. Por lo que respecta a la pasta básica de cocaína, la prevalencia de vida fue $1.9 \%$, mientras que la prevalencia de vida para uso de alucinógenos fue $0.5 \%$. También se reportó el uso de otras drogas ilegales como marihuana, cocaína y éxtasis, entre otras; siendo la edad promedio de inicio entre los 13 y 14 años. Algunos años después, Cedro (2017) reportó que, a nivel nacional (Perú), entre los jóvenes de 12 a 18 años, la prevalencia de vida de consumo de alcohol fue de $46.6 \%$ y la de pasta básica de cocaína, $0.2 \%$. Este informe reportó edades 
promedio de inicio más altas que las del informe de Devida, indicando que para el alcohol es de 18.3 años. El Consejo Nacional para el Control de Estupefacientes (Conace) (2007), institución del gobierno de Chile, señala que la prevención ideal es aquella que tiene como objetivo el mejoramiento de la calidad de vida, a través del desarrollo integral de la persona, ya que constituye el mejor protector para enfrentar cualquier situación de riesgo.

Los factores protectores y de riesgo no poseen necesariamente una relación causal con el consumo de SPA, sino más bien disminuyen o aumentan, respectivamente, la probabilidad de consumo (Conace, 2007). Los factores de protección son los que en constante interacción, pueden contribuir a reducir las probabilidades de que surjan problemas relacionados con el consumo de SPA; apoyando el desarrollo del individuo, (autoestima, tolerancia a la frustración, adecuada resolución de conflictos, etc. y factores sociales relacionados con un adecuado sistema de relaciones familiares, calidad en la comunicación, existencia de límites, promoción de estilos de vida saludables, redes de apoyo social, etc.). Asimismo, Nató y Rodríguez (2001) reportaron una diversidad de factores que pueden considerarse como de riesgo para el consumo y adicción a SPA. Entre ellos, están los factores personales (bajo nivel de autoestima, inconformismo con las normas sociales, depresión, etc.); factores familiares (clima familiar, normas de convivencia, etc.) y factores de carácter comunitario (dependencia del grupo, presión del grupo, resentimiento social, conflictos interpersonales, exceso de tiempo libre, disponibilidad de las drogas, etc.) Las motivaciones para el consumo más bien problemático (Conace, 2007) son el alivio de la tensión, la necesidad de aceptación por el grupo o la búsqueda de sensaciones.

Los centros de comunidad terapéutica, buscan propiciar cambios en la persona hallándoles un nuevo estilo de vida, rehabilitándolos en las distintas esferas personales y sociales, sin embargo durante el tiempo de experiencia de trabajo profesional en el tema de la adicción, se ha observado que existe mayores investigaciones y trabajos de prevención y promoción de salud referidos a la población adulta dejando algunas veces de lado a la población más joven que son los niños y adolescentes es por ello la necesidad de poder presentar investigaciones de rehabilitación en niños y adolescentes consumidores de sustancias psicoactivas. Al parecer, la primera comunidad terapéutica para adictos se inicia con el trabajo del psicoanalista alemán
Ernst Simmel en Berlín (Pérez, 2010). La idea era de disponer un espacio relativamente controlado en el que pudiera vigilarse con más cuidado la recuperación de los pacientes.

Son escasos los trabajos que investigan la calidad de vida en adictos. En un estudio de Anicama, Mayorga y Henostroza (2001), en Lima, investigaron en 67 sujetos de 21 a 41 años, adictos a SPA. Utilizaron la escala de calidad de vida de Olson y Howard y el cuestionario de recaídas para adictos de Marlat, aplicados ambos en forma individual. Hallaron que la calidad de vida de los adictos está muy por debajo de lo hallado en las poblaciones normales, es mejor en los hombres que en las mujeres y se deteriora conforme avanza la edad y el tiempo de consumo. Se observó que la cantidad de recaídas afecta negativamente la calidad de vida.

Aún más escasos son los estudios que investigan la calidad de vida relacionada con la salud en adictos. Lozano (2005) realizó en España un estudio con 359 pacientes de cinco comunidades terapéuticas gestionadas por la Fundación Andaluza para laAtención a las Drogodependencias. El estudio tuvo por finalidad primaria validar unos instrumentos, sin embargo, además, de ello, se detectaron diferencias en la calidad de vida relacionada con la salud (CVRS) en pacientes: 1) incluidos en programa de desintoxicación con la de otros que están en deshabituación, 2) consumidores de «rebujo» (mezcla de heroína y cocaína base) que están en programa de tratamiento con metadona con la de otros que no están en tratamiento con metadona, y 3) al ingreso en comunidad terapéutica y al finalizar el tratamiento.

Los estudios en adolescentes han explorado algunas variables parcialmente relacionadas con la calidad de vida. Por ejemplo, Zullig, Valois, Huebner, Oeltmann y Drane (2001) hallaron en adolescentes de Estados Unidos que los consumidores de SPA tenían un nivel de satisfacción con la vida más bajo que los no consumidores. De igual manera, Masferrer, FontMayolas y Gras (2012) compararon a adolescentes españoles consumidores de alcohol, tabaco o cannabis con no consumidores y hallaron que la satisfacción con la vida es más alta en adolescentes no consumidores que en los consumidores. No obstante, la satisfacción vital se entiende más como una evaluación global de la experiencia de vida que como un reporte del nivel de calidad en las áreas expresadas en las definiciones citadas más arriba. Por otro lado, si bien puede ser de interés realizar una comparación del nivel de calidad de vida relacionado con la salud entre consumidores y 
no consumidores, un enfoque diferente e igualmente productivo, podría consistir más bien en determinar cómo el nivel de calidad de vida relacionada con la salud podría estar asociado con el tiempo de consumo o con el tiempo de permanencia en la comunidad terapéutica. En el presente estudio, el tiempo de consumo de sustancias psicoactivas se define como el número de meses que tiene la persona como historia de consumo antes de que ingrese a un centro de rehabilitación, en tanto que el tiempo de permanencia se entiende como el número de meses de permanencia en la comunidad terapéutica. Se puede inferir que a mayor tiempo de consumo, la calidad de vida será menor, en tanto que los resultados del impacto del tiempo de permanencia en la comunidad terapéutica dependerán de muchos factores, asociados a la experiencia de la permanencia.

\section{MÉTODO}

\section{Participantes}

Los participantes fueron 86 , entre niños, niñas y adolescentes de ambos sexos de una comunidad terapéutica en la ciudad de Lima (Perú). Todos ellos eran de nivel socioeconómico bajo y sus edades se encontraban entre los 7 y los 18 años. Las drogas que consumían eran alcohol, terokal y pasta básica de cocaína. El centro de rehabilitación en el que estos niños, niñas y adolescentes residen tiene características de residencia familiar, con normas de convivencia y con actividades recreativas en familia.

\section{Instrumentos}

Test para la Evaluación de la Calidad de Vida en Adictos a Sustancias Psicoactivas (TECVASP) (Lozano, 2005).

Está formado por 22 ítems (18 positivos y 4 negativos [ítems 15, 19, 20 y 21]) con un formato de respuesta graduada de cinco alternativas. Las alternativas de respuesta son codificadas con las siguientes puntuaciones: a) en los ítems positivos: nada (5 puntos), poco (4 puntos), a veces (3 puntos), bastante ( 2 puntos) y mucho (1 punto); b) en los ítems negativos: nada (1 punto), poco (2 puntos), a veces (3 puntos), bastante (4 puntos) y mucho (5 puntos). De esta forma, en cada ítem una mayor puntuación representa una valoración más positiva del contenido, y en el test una mayor puntuación representa una mejor CVRS. El cálculo de la fiabilidad se realizó mediante el coeficiente de consistencia interna alfa de Cronbach (alfa =.861). El análisis de los ítems mostró valores del índice de homogeneidad (correlación ítemtotal corregido) aceptables para todos los ítems, a excepción de los obtenidos en los ítems $15(\mathrm{r}=0,022)$, $19(r=0,124)$ y $21(r=0,001)$, en los que se obtienen valores bajos.

Este instrumento se trabajó originalmente en adultos, por lo que fue necesario realizar algunos ajustes a los ítems para la investigación con niños y adolescentes. Se realizó el estudio piloto con una población de 168 estudiantes de un colegio de la ciudad de Lima, para establecer la validez y confiabilidad del instrumento, teniendo en cuenta los fines del trabajo de investigación en niños y adolescentes, de ambos sexos de la ciudad de Lima. Se realizó un estudio de jueces para determinar la validez lingüística. La confiabilidad de las puntuaciones se estableció mediante el alfa de Cronbach, que alcanzó el valor de .70. Luego se revisó las puntuaciones para caracterizar los niveles de calidad de vida para el presente estudio en niños y adolescentes de ambos sexos, estableciendo los cuartiles de la siguiente forma; de 0 a 82 puntos, nivel bajo; de 83 a 87 puntos, nivel regular; de 88 a 91 puntos, nivel bueno y de 92 a 112, nivel muy bueno.

\section{Procedimiento}

Se estableció contacto con la comunidad terapéutica a través de la dirección técnica de la comunidad, para la autorización del estudio. Se presentó el proyecto de investigación al Comité de Ética de la Universidad Peruana Cayetano Heredia, quienes autorizaron que a partir del 16 de Agosto del 2016, se inicie el trabajo de investigación. La dirección técnica y los familiares tutores de los participantes firmaron el documento de consentimiento de la investigación; así mismo los participantes firmaron el respectivo asentimiento. Finalmente, se realizó una actividad de intervención para elevar la motivación del cambio de vida teniendo en cuenta los resultados obtenidos.

\section{Análisis de datos}

Se calculó primero la proporción de participantes, por muestra total y por género, ubicados en cada una de las cuatro categorías o niveles de la CVRS. También se hallaron las tres medida de tendencia central (media, mediana y moda) del tiempo de consumo y tiempo de permanencia en la comunidad terapéutica, igualmente para la muestra total y por género. Finalmente se calcularon correlaciones bivariadas Spearman entre las tres variables (CVRS, tiempo de consumo y tiempo de permanencia). En todos los análisis se usó 
el SPSS15 y para las correlaciones se asumió un nivel de significación de 0,05 .

\section{RESULTADOS}

En la Tabla 1 se presenta la distribución de la muestra según edad, sexo y tipo de consumo. Teniendo en cuenta la definición de Infancia Media (611 años) y Adolescencia (11-19 años) según Papalia, Olds y Feldman (2010) se aprecia también que 10 participantes son infantes $(11,6 \%)$ y $76(88,4 \%)$ son adolescentes. También puede observarse que todos los participantes consumen ambos tipos de drogas.
En la tabla 2 se muestra los niveles de calidad de vida en la muestra general y según el sexo del participante. Lo más destacable es que en ambos sexos, la mayoría de participantes presentan un nivel bajo de calidad de vida. En el grupo femenino la situación es algo más benigna, incluso más del $20 \%$ reportan un nivel bueno o muy bueno de calidad de vida.

En la Tabla 3, se muestran las medidas de tendencia central del tiempo de consumo en meses. Se aprecia que la media del tiempo de consumo es ligeramente más alto entre las mujeres; la duración más frecuente es ambos es la misma. La mayor parte de los residentes

Tabla 1. Distribución de la muestra según edad, sexo y tipo de droga consumida

\begin{tabular}{clcccc}
\hline \multirow{2}{*}{ Edad } & \multicolumn{2}{c}{ Sexo } & & \multicolumn{2}{c}{ Tipo de droga } \\
\cline { 2 - 3 } \cline { 5 - 6 } & Masculino & Femenino & & Terokal & Pasta Básica \\
\hline 7 & 1 & 0 & & 1 & 1 \\
8 & 2 & 0 & & 2 & 2 \\
9 & 0 & 1 & & 1 & 1 \\
10 & 1 & 0 & & 1 & 1 \\
11 & 4 & 1 & & 5 & 5 \\
12 & 6 & 2 & & 8 & 8 \\
13 & 12 & 4 & & 16 & 16 \\
14 & 13 & 8 & & 21 & 21 \\
15 & 7 & 9 & & 16 & 16 \\
16 & 0 & 11 & & 11 & 11 \\
17 & 1 & 2 & & 3 & 3 \\
18 & 0 & 1 & & 1 & 1 \\
Total & 47 & 39 & & 86 & 86 \\
\hline
\end{tabular}

Tabla 2. Niveles de calidad de vida en la muestra total y según sexo

\begin{tabular}{lcccccc}
\hline Calidad de vida & M & $\mathbf{\%}$ & $\mathbf{F}$ & $\mathbf{\%}$ & Total & $\mathbf{\%}$ \\
\hline Baja & 38 & 80.85 & 28 & 71.79 & 66 & 77 \\
Regular & 1 & 4.26 & 2 & 5.13 & 3 & 3 \\
Buena & 5 & 10.64 & 2 & 5.13 & 7 & 8 \\
Muy Buena & 3 & 6.38 & 7 & 17.95 & 10 & 12 \\
Total & 47 & 100 & 39 & 100 & 86 & 100 \\
\hline Nota: $\mathrm{M}=$ masculino, $\mathrm{F}=$ femenino. & & & & &
\end{tabular}

Tabla 3. Medidas de tendencia central de tiempo de consumo de drogas (meses)

\begin{tabular}{lccc}
\hline & M & F & Total \\
\hline Media & 13.49 & 15.31 & 14.31 \\
Mediana & 12 & 12 & 12.00 \\
Moda & 12 & 12 & 12 \\
$\mathrm{n}$ & 47 & 39 & 86 \\
\hline Nota: &
\end{tabular}

Nota: $\mathrm{M}=$ masculino, $\mathrm{F}=$ femenino. 
está concentrada en un tiempo de consumo menor a los 20 meses.

En la tabla 4 se presenta las medidas de tendencia central del tiempo de permanencia en la comunidad terapéutica. Como se ve, la media de permanencia es algo más alta en el grupo femenino. La mediana y la moda también indican un mayor período de permanencia en las mujeres. En ambos casos, existe una proporción importante de residentes que tienen 10 meses o menos de permanencia.

Finalmente, tanto el tiempo de consumo como el de permanencia correlacionan negativamente con la calidad de vida. La correlación entre tiempo de consumo y calidad de vida fue $\mathrm{r}=-0,285(p=0,008) \mathrm{y}$ la correlación entre tiempo de permanencia y calidad de vida fue $r=-0,410(p<0,001)$. En suma, la calidad de vida es menor cuanto más tiempo de consumo o más tiempo de permanencia en la comunidad terapéutica presenta el niño o adolescente. La correlación negativa entre tiempo de consumo y calidad de vida es comprensible, dado que, el involucramiento en el consumo genera deterioros en diversos indicadores de salud. En cambio, llama la atención la correlación igualmente negativa entre el tiempo de permanencia y la calidad de vida, pero probablemente esto tiene su explicación en el hecho de que en este grupo en

Tabla 4. Medidas de tendencia central del tiempo de permanencia en la comunidad terapéutica (meses)

\begin{tabular}{lccc}
\hline & M & F & Total \\
\hline Media & 7.53 & 10.05 & 8.67 \\
Mediana & 5.00 & 9.00 & 6 \\
Moda & 1 & 2 & 2 \\
$\mathrm{n}$ & 47 & 39 & 86 \\
\hline
\end{tabular}

Nota: $\mathrm{M}=$ masculino, $\mathrm{F}=$ femenino.

particular, el tiempo de consumo y el tiempo de permanencia correlacionan positivamente $(\mathrm{r}=0,396$; $p<0,001)$. Probablemente, estos dos últimos resultados tienen que ver con la lentitud y dificultad generalmente observadas en los procesos de rehabilitación del abuso de sustancias.

\section{DISCUSIÓN}

En resumen, los resultados indican primero, la presencia de niños entre los consumidores; en segundo lugar, bajos niveles de CVRS en ambos sexos, aunque ligeramente más bajos en los hombres y en tercer lugar, que la CVRS correlaciona negativamente tanto con el tiempo de consumo, como con el tiempo de permanencia.
Un estudio realizado en el Perú por Devida (2013) mostró que la edad de inicio de consumo más baja se ubica en los 8 años, dato parecido a lo hallado en este trabajo, en el que se pudo ubicar al menos un niño de 7 años ya consumidor. Asimismo, en el estudio de Cedro (2017) se encontró que adolescentes de 12 a 18 años tenían una prevalencia de vida (usó alguna vez en la vida) de $46,6 \%$ en el caso del alcohol, sustancia que fue con la que se iniciaron en el consumo de drogas los participantes del presente estudio. Nos encontramos, por tanto, frente a una realidad en la que desde una edad temprana (7 u 8 años) muchos niños en el Perú ya están expuestos al consumo de sustancias altamente tóxicas, como el terokal y la pasta básica de cocaína, lo que, a la larga, tendrá consecuencias sociales de gran impacto negativo, incluyendo la reproducción de este patrón de comportamiento y un aumento en general de la problemática social relacionada con el consumo de sustancias (delincuencia, embarazos no deseados, replicación del ciclo de pobreza, etc.)

La investigación realizada por Casas y Bello (2012) dio a conocer que los niños del primer año de secundaria de colegios públicos y privados de España se encontraban muy satisfechos con sus vidas. Aparentemente en el estudio, esta población no indica que sean consumidores de alguna sustancia psicoactiva. Por otro lado, Casas et al., (2015) investigaron cómo era la calidad de vida de los niños latinoamericanos, hallando que ésta era mejor en comparación a los adultos; sin embargo el estudio se hizo en solo dos países de la región. Estos resultados están referidos a la población en general. Nuevamente, no existen estudios que comparen directamente el nivel de $\mathrm{CV}$ en general, ni CVRS en particular, entre niños no adictos y adictos a sustancias, si bien, en el caso de adultos, se sabe que la CVRS en adictos suele estar entre las más bajas en comparación con otros grupos poblacionales (e. g., Lizán, Reig, Richart \& Cabrero, 2000).

Por otro lado, el hecho de que la calidad de vida dentro de la comunidad terapéutica es menor cuanto mayor haya sido el tiempo de consumo podría explicarse en parte factores de adaptación y también, principalmente, por los propios efectos del historia de consumo de SPA. Los niños, niñas y adolescentes del presente estudio tienen arraigado el consumo a las diferentes SPA, ya que, antes de su ingreso en la comunidad terapéutica vivían en la calle, compartiendo lugares de riesgo al consumo de drogas y sin ninguna regla de convivencia o algo parecido a la normas de convivencia en casa. Al parecer, los resultados estarían indicando que cuanto mayor haya sido el tiempo de 
consumo antes del ingreso a la comunidad terapéutica, y quizá debido al deterioro que esto conlleva, los niños, niñas y adolescentes del estudio no muestran una adaptación total al medio donde residen actualmente, al encontrarse restringidos frente al consumo de drogas y de otras actividades ilícitas. Como ya se indicó, la comunidad terapéutica está organizada y estructurada como residencia familiar con sus respectivas normas de convivencia, un medio al que es difícil adaptarse tras un historial de consumo de SPA. Cabe especular que estas dificultades de adaptación tengan algún impacto en la CVRS, sin embargo, tal como se indicó, no debe dejarse de considerar las secuelas que un largo tiempo de consumo tiene en la propia salud.

El otro resultado correlacional indica que la CVRS disminuye con el tiempo de permanencia. Es decir, cuanto mayor el tiempo de permanencia, disminuye la CVRS. Una posible explicación recurriría nuevamente a la dificultad de adaptación ya mencionada, dificultad que, al parecer, no disminuye con el tiempo de permanencia. Este resultado parece, sin embargo, algo particular de esta población, considérese la correlación positiva entre tiempo de consumo y tiempo de permanencia, un resultado que no necesariamente existe en cualquier otra población. Es decir, es posible que, en la población investigada, la correlación negativa entre CVRS y tiempo de permanencia esté reflejando el hecho de que los sujetos con mayor tiempo de permanencia sean también los más deteriorados, es decir, con mayor tiempo de consumo. Algunos estudios han puesto de relieve los beneficios de un tratamiento en comunidad; por ejemplo, este tipo de intervención puede mejorar algunos índices de socialización como la tolerancia, responsabilidad y autonomía (Olivar \& Pedrero, 2004). Sin embargo, la mayoría de los estudios han sido llevados a cabo en adultos y por tanto sólo pueden brindar una idea aproximada de los factores que operan en el proceso de cambio dentro de una comunidad terapéutica cuando se trata de niños y de adolescentes. En todo caso, los resultados obtenidos deben ser motivo de un análisis ulterior respecto de los beneficios que puedan ser brindados en una comunidad terapéutica respecto, específicamente de la CVRS, pues, al parecer, este aspecto no está siendo suficientemente atendido en las acciones de rehabilitación y re-socialización de algunas de estas comunidades.

En esta investigación, más allá de dar resultados numéricos y cualitativos, se busca motivar a los demás profesionales a estudiar a una población vulnerable, como la de los niños, niñas y adolescentes, por tratarse de personas en desarrollo y que en ellos podemos proyectar el futuro del país. Finalmente, como posibles limitaciones del estudio señalaremos el haberse trabajado con una muestra relativamente pequeña y con un instrumento adaptado, siendo necesario el desarrollo de medias especialmente diseñadas para la población infantil y adolescente.

\section{REFERENCIAS BIBLIOGRAFICAS}

Anicama, J., Mayorga, E. \& Henostroza, C. (2001). Calidad de vida y recaídas en adictos a sustancias psicoactivas de Lima Metropolitana. Psicoactiva, 19, 7-27.

Berzon, R., Hays, R. D. \& Shumaker, S. A. (1993). Preface. International use, application and performance of health-related quality of life instruments. Quality of Life Research, 2, 367-368.

Breslin, S. (1991). Quality of life: How is it measured and defined? Urologia Internationals, 46, 246-251

Casas, F., Alfaro, J., Sarriera, J.C., Bedin, L., Grigoras, B., Bălţătescu, S., Malo, S. \& Sirlopú, D. (2015). El bienestar subjetivo en la infancia: Estudio de la comparabilidad de 3 escalas psicométricas en 4 países de habla latina. Psicoperspectivas,14(1),6-18. Recuperado de: http://www.psicoperspectivas. cl.doi:10.5027/psicoperspectivas-vol14- issue1fulltext-522

Casas, F \& Bello, A. (2012). Calidad de Vida y Bienestar Infantil Subjetivo en España. ¿Qué afecta al bienestar de niños y niñas españoles de $1^{\circ}$ de ESO? Madrid: UNICEF España.

CEDRO (2017). Epidemiología de drogas en población urbana peruana: Encuesta de hogares. Lima: CEDRO.

Comisión Nacional para el Desarrollo y Vida sin Drogas. (2013). IV Estudio Nacional:

Prevención y Consumo de Drogas en Estudiantes de Secundaria 2012. Lima: Devida-OPD.

Consejo Nacional para el Control de Estupefacientes CONACE \& Ministerio del Interior y Salud Pública (2007). Orientaciones Generales para la detección precoz del consumo de drogas. Santiago de Chile: Gobierno de Chile.

Corless, I. B., Nicholas, P. K. \& Nokes, K. M. (2001). Issues in cross-cultural quality of life research. Journal of Nursing Scholarship, 33, 15-21.

Lizán, L., Reig, A., Richart, M. \& Cabrero, J. (2000). Evaluación de la calidad de vida relacionada con la salud mediante las láminas COOP/WONCA. Medicina Clínica (Barcelona), 114 (Supl. 3), 76-80, 2000.

Lozano, O. M. (2005). Construcción de un test para medir la calidad de vida relacionada con la salud 
en drogodependientes. Aplicación de un modelo politómico de la teoría de respuesta al item. (Tesis Doctoral. Universidad de Granada, Granada, España). Masferrer, L., Font-Mayolas, S. \& Gras, M.E. (2012). Satisfacción con la vida y consumo de sustancias psicoactivas en la adolescencia. Cuadernos de Medicina Psicosomática y Psiquiatría de Enlace, 102, 47-53.

Nató, A. \& Rodríguez, G. (2001). Las víctimas de las drogas. Buenos Aires: Editorial Universidad.

Olivar, A. \& Pedrero, E. J. (2004). Evaluación de la intervención socioeducativa en comunidad terapéutica: estudio de una muestra mediante el CCE-R. Trastornos Adictivos, 6, 46-60.

Organización Mundial de la Salud (1969). Comité de Expertos de la OMS en Farmacodependencia. (Serie de Informes Técnicos N407). Ginebra: Organización Mundial de la Salud.

Papalia, D. E., Olds, S. W. \& Feldman, R. D. (2010). Desarrollo humano. Ciudad de México: McGrawHill.
Pérez, F. (2010). Origen alemán de la comunidad terapéutica. Revista de la Asociación Española de Neuropsiquiatría, 30, 145-149.

Stuifbergen, A.K., Seraphine, A. \& Roberts, G. (2000). An explanatory model of health promotion and quality of life in chronic disabling conditions. Nursing Research, 49, 122-130.

Wilson, M. \& Cleary, P. (1995). Linking clinical variables with health related quality of life. JAMA, 273(1), 59-65

World Health Organization Quality of Life, Group. (1995). The World Health Organization Quality of Life Assessment (WHOQOL): Position paper from the World Health Organization. Social Science and Medicine, 41(10), 1403-1409.

Zullig, K.J., Valois, R.F., Huebner, E.S., Oeltmann, J. E. \& Drane, J. W. (2001). Relationship between perceived life satisfaction and adolescents' substance abuse. Journal of Adolescent Health, 29, 279-288. 